\title{
Ökonomisierung der Wissenschaft
}

\section{Peter Weingart}

Die Universität entwickelt sich immer rascher von ihrer traditionellen Rolle, Hort des Wissens als moralische und kulturelle Kraft zu sein, geschützt von der sie umgebenden weltlichen Gesellschaft, hin zu einem zentralen Element des ,nationalen Innovationssystems' (David 1997: 3, 5; Etzkowitz/Leydesdorff 1998). Ein neuer ,Gesellschaftsvertrag‘ zwischen der Universität und der Gesellschaft mit wesentlich spezifischeren Konditionen als zuvor werde ausgehandelt. Diese und ähnliche Einschätzungen, manche euphorisch, andere bedauernd und warnend, charakterisieren eine fundamentale Veränderung im wissenschaftspolitischen Diskurs allgemein und eine Perspektivverschiebung bezüglich der Rolle der Universität im Besonderen. Wenn es zutrifft, dass wissenschaftliches Wissen tatsächlich in einem umfassenden Verständnis zur Ware wird, dann wären damit tiefgreifende Veränderungen der sozialen Werte und der institutionellen Grundlagen angezeigt, auf denen das gegenwärtige Wissenschaftssystem beruht. So würde die allgemeine öffentliche Verfügbarkeit über Wissen aufgegeben und an ihre Stelle träten Eigentumsrechte an Wissen. Sie machen den Handel mit der Ware Wissen erst möglich und lassen Geheimhaltung sowie strategische Verwendung zu. Wo Wissen zur Ware wird, muss sich die Vertrauensbasis des Wissens ändern: Vertrauen erhält nur der demonstrierte Nutzen. Die Spekulation um ihrer selbst willen hätte keinen Platz mehr, es sei denn, sie würde mit, venture capital' ausgestattet, weil sie einen zukünftigen Ertrag verspräche.

Bislang besteht eine Spaltung der Wissenskulturen: in eine akademische, in der die Produktion des Wissens durch die Gesellschaft gefördert wird und der Zugang zu ihm frei ist, und in eine kommerzialisierte, in der das Wissen aufgrund seines wirtschaftlichen Nutzens mit Eigentumsrechten versehen auf Märkten gehandelt wird. Die ,Kommodifizierung' und ,Kapitalisierung wissenschaftlichen Wissens ist also nicht grundsätzlich neu. Die Frage ist, ob den Befürchtungen eine Entwicklung zu einer bedrohlichen oder gar vollkommenen Verwandlung des Wissens zur Ware zugrundeliegt und damit die akademische Ordnung der Wissensproduktion nachhaltig verändert wird. Die Problematik der Ökonomisierung der Wissenschaft wirft zunächst zwei 
allgemeine Fragen auf: Verändert sich das Verhältnis zwischen Wissenschaft (Universität) und Industrie und welche Folgen haben die Veränderungen für die Wissenschaft? Ergeben sich damit auch Folgen für den Wahrheitscharakter und die Verlässlichkeit wissenschaftlichen Wissens?

\section{Was kann Ökonomisierung heißen?}

Die bekannte These, dass die Ökonomisierung die Wissenschaft gefährde, wird nur dann verständlich, wenn man zwischen beiden einen inhärenten Gegensatz konstruiert. Ich werde dagegen auf historische Phasen verweisen, in denen das nicht der Fall war. Was sind also mögliche Aspekte eines Gegensatzes zwischen Wissenschaft und Ökonomie? Wie werden dabei die Wissenschaft und wie die Ökonomie implizit oder explizit charakterisiert?

Die übliche Gegenüberstellung ist die zwischen Grundlagenforschung und angewandter Forschung. Die Unterstellung ist, dass Grundlagenforschung gleichsam der Normaltypus von Wissenschaft ist: zwar nur einer relativ kleinen Elite (den akademischen Forschern in den Universitäten) vorbehalten, aber von so zentraler Bedeutung für die gesamte Wissenschaftsentwicklung, dass sie Basis und Kern in einem ist. Angewandte Forschung wäre demgegenüber durch pragmatische Nutzenorientierung charakterisiert. Dahinter verbergen sich unterschiedliche Steuerungsmodelle der Wissensproduktion. Im Modell der Grundlagenforschung wird die Entwicklung wissenschaftsintern durch die Disziplinen gesteuert. Im Gegensatz dazu wird in der angewandten (ökonomisierten, politisierten oder militarisierten) Forschung die Relevanz von Fragestellungen von außen vorgegeben.

Ist der Gegensatz wirklich so scharf? Man könnte zum Beispiel argumentieren, dass Förderprogramme jeder Art, die thematische Vorgaben machen, denselben Effekt der Selektivität haben. Außerdem wird die Wissenschaft selbstverständlich in einem gesellschaftlichen Kontext betrieben. Daraus erwachsen ihr Präferenzen, Fragestellungen und unterschiedliche Interessen, die sie nicht vollkommen ignorieren kann - und auch faktisch nicht ignoriert. Jede Wissenschaftspolitik ist gezwungen, Prioritäten zu setzen, um knappe Mittel zu verteilen. Wenn die Wirtschaft als ein wesentlicher Finanzier von Forschung gemäß ihren Bedürfnissen und Interessen bestimmten Forschungsgebieten und Forschungsfragen eine höhere Priorität einräumt als anderen, dann handelt es sich nicht um eine Ökonomisierung der Wissenschaft, sondern um bloße selektive Prioritätensetzung aus Sicht der Wirtschaft.

Etwas anders stellt sich das Problem dar, wenn der Gegensatz zwischen Erkenntnis- und Nutzenorientierung aufgemacht wird. Der Wissenschaftstheoretiker Martin Carrier spezifiziert diesen Gegensatz dahingehend, dass die Nutzenorientierung eine andere epistemische Strategie nach sich ziehe 
(Carrier 2007: 93). Demzufolge bleibt das ,Naturverstehen' auf der Strecke, denn Nutzenorientierung leitet die Forschung jeweils nur solange, bis die betreffende Anwendung (eine Technik, ein Produkt oder Verfahren) vorliegt und an den Markt gebracht werden kann. Unter Umständen ist sogar die Qualität, das heißt die Verlässlichkeit des generierten Wissens betroffen. Ökonomisierung würde hier, wäre sie flächendeckend, auf der Grenze zwischen thematischer Selektivität und Qualitätsverlust wirksam werden. Dieser Fall mag durchaus relevant häufig vorkommen, aber er ist nicht zwangsläufig. Das Beispiel Pasteurs widerspricht etwa der Ausschließlichkeit von Nutzenorientierung und Naturverstehen, zumindest im Prinzip. Entscheidend ist hier, dass es bei den analytischen Unterscheidungen um die Motivation der Forschung geht (Nutzen oder Neugier). In der Realität kommt es dann etwa darauf an, wer die Forschung bezahlt und mit welchem Interesse. In den Pasteur'schen Forschungen über die Ursachen des Milzbrands war es notwendig, die Untersuchungen bis zur endgültigen Aufklärung voranzutreiben. Ließe sich die erwünschte Kontrolle über einen Effekt auch ohne die Kenntnis der Ursachen, zum Beispiel durch Simulation erreichen, würde ein ökonomisch denkender Finanzier auf die Ursachenerforschung verzichten. Vorstellbar ist zum Beispiel eine Arbeitsteilung zwischen öffentlich und privat finanzierter Forschung, in der die öffentliche Hand die Förderung der Naturerkenntnis verantwortet. Dies ist ohnehin faktisch der Fall.

\section{Jenseits der Rhetorik: öffentliche vs. private Wissenschaft}

Die dichotome Konzeptualisierung von Grundlagenforschung und angewandter Forschung als essentieller Gegensatz zwischen Wissenschaft und Ökonomie hat historische Wurzeln. Mirowski und Sent (2007) ziehen in ihrem Rückblick auf unterschiedliche Regime der Wissenschaftsorganisation in den USA den Schluss, dass die Rhetorik der freien und ,reinen' Grundlagenforschung ein ideologisches Konstrukt des Kalten Krieges ist, als das Militär zum mächtigsten und einflussreichsten Geldgeber für die Forschung wurde. In diesem Kontext wurde die Wissenschaft de facto den nationalen strategischen Zielen unterworfen, aber aus ideologischen Gründen als autonom und autark ausgegeben. Die ,reine' Grundlagenforschung wurde rhetorisch zum Synonym für Freiheit und Demokratie (ebd.: 654).

Schon spätestens seit Mitte des 19. Jahrhunderts hat es institutionalisierte Verbindungen zwischen Wissenschaft und Wirtschaft gegeben. Die sogenannte Grundlagenforschung ist nicht die einzige Form der Forschung, durch die Naturverstehen ermöglicht wird. Das heißt aber nicht, dass es nicht Veränderungen gibt, die sich als Ökonomisierung bezeichnen lassen und deren Auswirkungen auf die Wissenschaft es wert sind, untersucht zu werden. Diese Veränderungen betreffen vor allem die Kerninstitution der 
Wissenschaft, die Universität. Die Universität nimmt deshalb den zentralen Platz in der Wissenschaft ein, weil in ihr die Grundprinzipien der Wissenschaft ihren institutionellen Ort haben. Das heißt: Hier sind der Idee nach die Bedingungen institutionell gegeben, die für die Produktion wissenschaftlichen Wissens notwendig sind. Sind sie es nicht, bedeutet das nicht unbedingt den Zusammenbruch aller Forschung, aber die Glaubwürdigkeit und Verlässlichkeit des Wissens sind dann nicht in gleicher Weise gesichert. Das bedeutet, der Gesellschaft fehlt die Instanz ihres Lernens und ihrer Selbstvergewisserung. Was das bedeutet, ist an den Gesellschaften ablesbar, die nicht über ein leistungsfähiges Wissenschaftssystem verfügen. Das geringste Übel ist die mangelnde Innovationsfähigkeit und der damit entgangene materielle Wohlstand. Gravierender sind die defizitären gesellschaftlichen Infrastrukturen, angefangen von einem funktionierenden Regierungs- und Rechtssystem bis hin zu einem leistungsfähigen Bildungssystem sowie einer menschenwürdigen sozialen Infrastruktur. Welcher Art sind also die Bedingungen, die die Universität als Kerninstitution der Wissenschaft im Unterschied $\mathrm{zu}$ anderen Institutionen bietet?

Zumindest drei Grundbedingungen kennzeichnen die Idee der Universität und der in ihr betriebenen Forschung und Lehre: erstens, die primäre Orientierung an der Produktion (Forschung) und Verbreitung (Lehre) von Wissen beziehungsweise die unbedingte Priorität des Wahrheitskriteriums gegenüber dem Nutzenkriterium; zweitens, die Unabhängigkeit von anderen Interessen als denen an der Wissensproduktion selbst; und drittens, die Bewahrung der Wissensgenerierung und -verbreitung vor der Instrumentalisierung durch politische oder wirtschaftliche Interessen und die Sicherung der vorbehaltlosen Kommunikation in der Forschung und Lehre. Die Wissenschaft hängt vom organisierten Skeptizismus ab, der ebenfalls die Qualität des kommunizierten Wissens garantiert. Alle drei Prinzipien werden von keiner anderen Institution in einem Sozialisierungs- und Initiationsverfahren an die Mitglieder weitergegeben. Sie charakterisieren die Universität als Institution auch unabhängig davon, ob es sich, wie häufig in den USA, um privat finanzierte Einrichtungen oder um öffentliche Universitäten handelt. Wenn man diese Prinzipien so akzeptiert, entgeht man den Tücken des Gegensatzes zwischen reiner Grundlagenforschung und angewandter Forschung und kann präziser fragen, welche Effekte der Ökonomisierung mit ihnen in Konflikt stehen. Entscheidend dabei ist, ob die Prinzipien systematisch in Frage gestellt werden. Der Gegensatz, der damit aufgemacht wird, ist der zwischen öffentlicher und privater Wissenschaft. (Die Militärforschung als Sonderfall soll hier ausgenommen werden).

Es gibt zwar keine inhärente Beziehung zwischen der Wissenschaft und dem Allgemeinwohl einer Gesellschaft. Die Universität, so wie ich sie durch die Grundprinzipien charakterisiert habe, ist jedoch eine öffentliche Institution. Das heißt, sie ist allen Mitgliedern der Gesellschaft offen (auch wenn sie 
faktisch in bestimmten Gesellschaften etwa Frauen und Arbeiterkinder diskriminiert) und der Gesellschaft insgesamt gegenüber rechenschaftspflichtig. Mit Blick auf das in ihr produzierte Wissen bedeutet dies, dass grundsätzlich die Gesellschaft insgesamt Zugang dazu haben muss. Der Konflikt zwischen öffentlicher und privater Wissenschaft und damit die nicht triviale Tendenz zu einer Ökonomisierung der Wissenschaft lässt sich an einigen neuralgischen Punkten zeigen. Erstens wäre zu fragen: Wo und wie wird die Orientierung der Universität an der Wahrheit verändert? Zweitens, wo und wie wird die Interessenunabhängigkeit verletzt? Drittens, wo und wie wird die offene Kommunikation bedroht oder verhindert?

Ein Wandel der Orientierung der Universität am Wahrheitscode ist durch mehrere Dinge indiziert. Die Verlagerung der industriellen Forschung in die Universitäten hinein ist - bis auf die technischen Universitäten - bislang nur in wenigen Fällen in nennenswertem Umfang passiert. Wenn die finanzielle Abhängigkeit einer Universität von privaten Geldgebern (ausgenommen Stiftungen) zu groß wird, muss man davon ausgehen, dass es zu Interessenkonflikten kommt. Bislang ist diese Gefahr allerdings kaum gegeben, die krassen Fälle industrieller Forschungsverträge konzentrieren sich zudem auf spezifische Forschungsbereiche wie die Biomedizin, Molekularbiologie und Informatik. Aber die Gefahr, dass die Universitäten ihre ureigene Mission verraten, wird durch eine Reihe weiterer Faktoren begünstigt. So wird für die europäischen Universitäten ein substantieller Wandel der Forschungsziele konstatiert, der mit der erzwungenen Diversifizierung der Förderquellen einhergeht. Der Rückgang der öffentlichen Mittel wird zumindest zum Teil durch private, etwa industrielle Mittel, zu kompensieren versucht (Geuna/ Nesta 2006: 791). Die Zuwendungen aus der Industrie haben in aller Regel aber Verträge zur Folge, die den Firmen das Verwertungsrecht für die Forschungsergebnisse in Form von Patenten und Lizenzen einräumt. Hat die wachsende Zahl solcher Abkommen Auswirkungen auf die oben genannten Prinzipien? Die Forschungen dazu sind noch immer lückenhaft und unsicher, wobei die Situation in den USA besser bekannt ist als in Europa. Die Übersichten von Technologietransfer-organisationen sagen nichts über die Auswirkungen auf die akademische Forschung aus. Die vorrangige Frage ist immer die nach der Verzögerungswirkung von Patenten, die von zwei Dritteln der befragten Wissenschaftler bestätigt wird (ebd.: 797). Sie sagt aber noch nichts über die inhaltlichen Effekte der Patentierung.

Geuna und Nesta nennen unter anderen vier mögliche Gefahren eines intensivierten Engagements mit den Intellectual Property Rights (IPRs): erstens, die Prioritierung von Patenten gegenüber Publikationen, die insbesondere für jüngere Wissenschaftler einen Substitutionseffekt hat, weil sie dann weniger intellektuelles Kapital ansammeln und auf längere Sicht weniger produktiv sind; zweitens, eine negative Auswirkung auf die Kultur der offenen Wissenschaft in Gestalt zunehmender Geheimhaltung und des erschwerten 
Zugangs zu Forschungsmaterial; drittens, eine Gefährdung der Qualität der Lehre durch eine höhere Gewichtung von Patenten in den üblich werdenden Leistungsevaluationen. Schließlich bestünde die vierte Auswirkung in der Ablenkung von langfristigen grundlegenden Forschungsfragen zugunsten kurzfristiger. Das zeigt sich etwa in der Physik, in der die Trennung zwischen Grundlagen- und Anwendungsforschung möglich ist, aber nicht in der Biotechnologie. Unter diesen spekulativ postulierten Gefahren der Ökonomisierung der Wissenschaft durch Patentierung stellen nur die Gefährdung der Kultur der offenen Wissenschaft und die kurzfristige Orientierung der Forschung wirklich einen Konflikt mit den Grundprinzipien der Universität dar.

Die befürchtete Veränderung des Zeithorizonts der privaten Forschung im Vergleich zur öffentlichen Forschung wird empirisch tatsächlich für die biotechnologische Forschung beobachtet. Hier ist allerdings sorgfältig zwischen der nostalgischen Klage der Wissenschaftler über den Verlust ihrer Freiheit und den wirklich nachteiligen Folgen für das System Wissenschaft zu unterscheiden. Außerdem ist zu klären, wie flächendeckend der postulierte Effekt ist. Es deutet alles darauf hin, dass die Patentierung in relevantem Umfang weitgehend auf die Bio- und Kommunikationstechnologie beschränkt, also auf die Spezifität des Wissens in diesen Gebieten zurückzuführen ist.

Eine weitergehende Frage ist, ob über die Patentierung das Verwertungsinteresse der Forscher das Erkenntnisinteresse verdrängt. Das führt letztlich zur Gefährdung der ,Kultur der offenen Wissenschaft ${ }^{\prime}$ und damit zum Kern der Gefährdung der Wissenschaft durch Ökonomisierung. Die Verzögerung der Kommunikation durch IPRs ist vergleichsweise trivial gegenüber einer solchen dauerhaften Behinderung. Die biomedizinischen Gebiete sind wiederum das vorrangige Beispiel für eine vergleichsweise neue Form der Ökonomisierung, nämlich der Belegung von Forschungsinstrumenten und Daten mit IPRs. Dies wird seit einiger Zeit mit den sogenannten Material Transfer Agreements (MTAs) betrieben, Nutzungsverträgen, die zwischen öffentlichen oder privaten Forschungseinrichtungen über die Verwendung bestimmter Instrumente, Proben oder Daten abgeschlossen werden. Zwar wird von den Verteidigern dieser Entwicklung angeführt, dass mit den formalen Verträgen, ähnlich wie bei Patenten, die Nutzungsmöglichkeiten erweitert, ja sogar die Entwicklung neuer Instrumente finanziert werde. Die Gegner verweisen jedoch auf die immer komplizierter werdenden Vertragswerke, die Kosten und die indirekten Effekte der Abschreckung von Forschern bis hin zur Verweigerung von Forschungskooperationen (Mirowski 2008). Obgleich sich Ökonomen und Wissenschaftler in ihren Interpretationen der Auswirkung von MTAs und Patenten widersprechen, laufen die Befunde auf dasselbe hinaus: Gravierender noch als die effektiven Störungen des wissenschaftlichen Kommunikationsprozesses ist die Ökonomisierung (und mit ihr verbunden die Verrechtlichung) der akademischen Kommuni- 
kation. Sie verstößt gegen alle drei der genannten Prinzipien. Die Orientierung der Kommunikation - erzwungen durch die rechtliche Sanktionierung - am Profitkriterium muss, wenn sie sich im Wissenschaftssystem ausbreiten würde, längerfristig fatale Folgen für das Wissenschaftssystem haben. Die Kommunikation würde dann monetären Kalkülen unterworfen, die die Verlässlichkeit des Wissens nachhaltig gefährden würden. Die entsprechenden Effekte sind nicht leicht kausal zu erfassen, weil sie indirekt und allmählich auftreten.

\section{Wandel der akademischen Kultur}

Es handelt sich um Veränderungen der Kultur der Wissenschaft, die zuallererst in den Bereichen sichtbar werden, in denen die Patentierung bereits in größerem Umfang praktiziert wird, es um viel Geld geht und öffentliche und private Förderung enger ineinander greifen als in anderen Forschungsgebieten: Biomedizin, klinische Tests, Pharmaforschung. Dort ist auch die Inzidenz von Betrugsfällen am höchsten. Ihr gehäuftes Auftreten hat die institutionalisierte Wissenschaft aufgeschreckt und dazu veranlasst, Kodizes guten wissenschaftlichen Verhaltens zu verkünden (so DFG und MPG). Es herrscht unter Wissenschaftlern selbst schon eine gewisse Nonchalance gegenüber dem hergebrachten Ethos. „Warum sollten Wissenschaftler ihre Daten nicht beschönigen, wie es in den Medien oder der Werbebranche üblich ist und von der Öffentlichkeit auch vorausgesetzt wird? [...] Warum sollte ein Forscher nicht erfolgreiche Ideen seiner Kollegen kopieren, ohne ihre Urheber zu nennen, wenn dies in marktorientierten Handlungsbereichen ein möglicher Weg zum Profit ist?" (Weingart 1998) So oder ähnlich könnten sich die Wissenschaftler fragen, bevor sie den entscheidenden Schritt tun, und sie wären in dem Augenblick der Beleg dafür, dass sich der Code der Ökonomie über den der Wissenschaft geschoben hätte. Die Zahlen sind beunruhigend, die Art des Umgangs mit den Betrugsfällen ist es auch. Sie werden sowohl von den Medien als auch von der Wissenschaft individualisiert, also als Verfehlungen von Individuen wahrgenommen, von ,schwarzen Schafen', die es in allen Bereichen der Gesellschaft gibt (Franzen et al. 2007).

Es wird nicht gesehen, dass es sich um die Warnzeichen eines strukturellen Wandels der akademischen Kultur handelt. Es ist in der Tat unerheblich, ob ein paar Forscher auf der Jagd nach dem Glück kriminelle Energie entwickeln. Es ist aber nicht unerheblich, wenn durch eine sich stetig ausweitende Ökonomisierung die wichtigste Ingredienz wissenschaftlichen Wissens abhanden kommt: das Vertrauen in jenes Wissen, das sich der ausschließlichen Orientierung an der Wahrheit verdankt. Es ist nur in öffentlichen Institutionen zu haben. 


\section{Literatur}

Carrier, Martin, 2007. Erkenntnisgewinn und Nutzenmehrung. In: Peter Weingart, Martin Carrier und Wolfgang Krohn, Nachrichten aus der Wissensgesellschaft. Analysen zur Veränderung der Wissenschaft. Weilerswist: Velbrück Wissenschaft, 93-110.

David, Paul, 1997. The Knowledge Factory. The Economist. A Survey of Universities. 4. Oktober, $1-22$.

Franzen, Martina/Rödder, Simone/Weingart, Peter, 2007. Fraud. Causes and Culprits as Perceived by Science and the Media. EMBO reports, 8, 1, 3-7.

Etzkowitz, Henry/Leydesdorff, Loet, 1998. The Endless Transition. A “Triple-Helix” of University-Industry-Government Relations. Minerva, 36, 203-208.

Geuna, Aldo/Nesta, Lionel J.J., 2006. University Patenting and its Effects on Academic Research. The Emerging European Evidence. Research Policy, 35, 790-807.

Mirowski, Philip, 2008. Livin' with the MTA. Minerva, 46, im Druck.

Mirowski, Philip/Sent, Esther-Mirjam, 2007. The Commercialization of Science and the Response of STS. In: Edward Hackett u.a., Hg., The Handbook of Science and Technology Studies. Thousand Oaks/London: Sage, 635-689.

Weingart, Peter, 1998. Ist das Wissenschaftsethos noch zu retten? Gegenworte, 2, 13-17.

\section{Peter Weingart}

Institut für Wissenschafts- und Technikforschung

Universität Bielefeld

PF 100131

D-33501 Bielefeld

E-Mail: weingart@uni-bielefeld.de 